\title{
Rupture of a Type B Aortic Dissection in a Postpartum Patient with Marfan Syndrome
}

\author{
Elizabeth Patberg, MD ${ }^{1}$ Jennifer Duffy, MD ${ }^{1}$ Afshan B. Hameed, MD ${ }^{1}$ \\ ${ }^{1}$ Department of Obstetrics and Gynecology, Irvine Medical Center, \\ University of California, Orange, California \\ Address for correspondence Elizabeth Patberg, MD, Department of \\ Obstetrics and Gynecology, University of California, Irvine, 333 City \\ Blvd. West, Ste 1400, Orange, CA 92868 (e-mail: epatberg@uci.edu).
} Am J Perinatol Rep 2019;9:e256-e261.

\begin{abstract}
\section{Keywords}

- Marfan syndrome

- pregnancy

- aortic rupture

- Type B aortic dissection

Background Pregnant women with Marfan syndrome (MFS) are known to be at increased risk of aortic dissection; however, cases of aortic rupture are extremely rare. There is lack of consensus on the exact site and size of aortic diameter measurement that increases this risk, and whether this applies to both Type $A$ and Type $B$ dissections. Case A 23-year-old G2P1001 with known Marfan syndrome who underwent an uncomplicated antepartum and intrapartum course. She experienced persistent backache 10 days postpartum that led to the diagnosis of Stanford Type B dissection. The patient was hospitalized for close observation. Dissection progressed to aortic rupture within 24 hours that required emergent thoracic endovascular aortic repair. She had an uncomplicated postoperative course.

Conclusion Our report demonstrates rupture of a known aortic dissection within a very short time in the postpartum period. The case highlights the importance of patient education and close surveillance especially in the postpartum period. It also brings home the value of imaging of the whole aorta rather than focusing on the ascending alone. Multidisciplinary care and timely diagnosis and intervention likely led to the favorable outcome in our case.
\end{abstract}

Marfan syndrome (MFS) is an autosomal dominant condition affecting 1:3000 to 1:5000 individuals due to a mutation in the gene encoding the connective tissue protein fibrillin-1 (FBN-1). ${ }^{1}$ Affected individuals have a wide variety of phenotypic manifestations involving skeletal, renal, ocular, skin, and cardiovascular organ systems. Aortic root disease including aneurysm, regurgitation, and dissection accounts for the majority of the cardiovascular morbidity and mortality in MFS. This morbidity likely stems from the loss of elastic lamellae and smooth muscle cells in the medial layer of the aorta, leaving it more vulnerable to shearing forces and dynamic changes in circulatory pressures. ${ }^{2,3}$ The physiologic changes of pregnancy that make pregnant women with MFS

DElizabeth Patberg's ORCID is https://orcid.org/0000-0003-38436179 .

received

May 1, 2019

accepted after revision

May 14, 2019
DOI https://doi.org/

$10.1055 / \mathrm{s}-0039-1692712$. ISSN 2157-6998. more vulnerable to complications of aortic disease are primarily related to intimal shear forces due to increase in cardiac output and hormonally mediated connective tissue alterations that may cause weakness of the arterial wall., ${ }^{4,5}$ Hemodynamic changes are more pronounced in the third trimester and postpartum period which may explain the increased propensity of aortic complications during this time frame.

The majority of the literature regarding pregnancyrelated aortic dissection in MFS patients is limited to case studies, case series, or retrospective reviews, and therefore, the precise risk of aortic dissection in pregnancy remains unclear. Much of the data originates from studies in which the diagnosis of MFS was unknown during the index pregnancy. A literature review that included 1,142 pregnant patients with MFS demonstrated that the rate of

Copyright @ 2019 by Thieme Medical Publishers, Inc., 333 Seventh Avenue, New York, NY 10001, USA. Tel: +1(212) 584-4662.

\section{License terms}

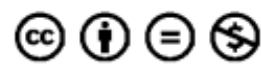


cardiovascular complications in was $2 \%$ to $6 \%$, ${ }^{6}$ which may be an overestimate due to selection bias. Investigators have attempted to stratify risk of dissection in pregnancy based on aortic root diameter. Risk of dissection has been reported to be $<1 \%$ if aortic root is $<40 \mathrm{~mm}^{7,8}$ and as high as $10 \%$ if $>40 \mathrm{~mm}^{9}$; however, evidence to support the latter claim is lacking. ${ }^{10,11}$ Nevertheless, the 2010 American College of Cardiology Foundation (ACCF/AHA) thoracic aortic disease guidelines recommend against pregnancy in women with MFS and an aortic root $>40 \mathrm{~mm}$, or for prophylactic repair in the event that they would like to become pregnant. ${ }^{12}$

Controversy exists regarding the aortic root diameter at which the risk of dissection and other cardiac complications becomes clinically significant. Based on prospective studies in which women were followed with serial aortic root measurements and were under the care of a cardiologist/ multidisciplinary team during pregnancy, the risk of aortic complications appears to be low, including when the aortic root is $>40 \mathrm{~mm}$ but less than $45 \mathrm{~mm} .^{7,11,13-16}$ Consistent with these findings, the 2014 European Society of Cardiology (ESC) guidelines on diagnosis and treatment of aortic disease $^{17}$ and the 2014 Canadian Cardiovascular Society position statement on the management of thoracic aortic disease, ${ }^{18}$ as well as the ESC guidelines for the management of cardiovascular diseases during pregnancy, ${ }^{19}$ recommend against pregnancy when the aortic root is $>45 \mathrm{~mm}$, or 41 to $45 \mathrm{~mm}$ in cases a rapidly enlarging aortic root or family history of dissection/sudden death.

We describe a case of an adverse pregnancy-related outcome in a woman with MFS whose aortic root was $<45 \mathrm{~mm}$ at the sinus of Valsalva and $<40 \mathrm{~mm}$ at the sinotubular junction, despite preconception planning, early establishment of prenatal care, and close monitoring with a multidisciplinary specialty team in a quaternary care facility.

\section{Case}

The patient is a 23-year-old G2P1001 who established prenatal care at our institution at 13 weeks of pregnancy. Her past medical history was significant for MFS diagnosed at age 12 years based on Revised Ghent Nosology. ${ }^{20}$ There was no family history of MFS, cardiac disease, or sudden death. She had a prior uncomplicated, term vacuum-assisted vaginal delivery approximately 2 years ago at our institution. The aortic root was stable throughout that pregnancy and postpartum period at $3.9 \mathrm{~cm}$. Postnatally, an FBN1 mutation was confirmed in both the patient and her newborn.

One month prior to conception in the currently described pregnancy, an echocardiogram at an outside hospital demonstrated an aortic root diameter of $42 \mathrm{~mm}$. Computed tomography (CT) angiogram of the chest confirmed this measurement and there were no ascending or descending aortic aneurysms or dissections noted. The abdominal aorta was not imaged. Repeat echocardiogram at our institution was performed at 17 weeks EGA that revealed aortic root of $42 \mathrm{~mm}$ at the sinus of Valsalva and $31 \mathrm{~mm}$ at the sinotubular junction. She was counseled on the risks of MFS in pregnancy and desired to continue the pregnancy. She was started on metoprolol $12.5 \mathrm{mg}$ twice daily at 16 weeks EGA and continued at this dose throughout her pregnancy. Her blood pressure remained within normal limits throughout pregnancy with systolic pressures 90 to $120 \mathrm{~mm} \mathrm{Hg}$ and diastolics of 50 to $80 \mathrm{~mm} \mathrm{Hg}$. A repeat echocardiogram was performed at 28 weeks EGA demonstrating an aortic root measurement of $41.8 \mathrm{~mm}$ at the sinus of Valsalva and 37.6 at the sinotubular junction. The remainder of her prenatal course was unremarkable. At 37 weeks 1 day EGA she underwent an uncomplicated vacuum-assisted vaginal delivery of a 3,140 g male infant with 1 - and 5-minute APGAR scores of 8 and 9, respectively.

The patient was discharged to home 2 days after an uncomplicated postpartum hospital stay. On postpartum day 5 she presented to an outside hospital emergency department with back pain. A noncontrast CT was unremarkable and she was discharged to home with treatment for a urinary tract infection. On postpartum day 10 , she represented to an outside emergency department with severe mid-to-upper back pain, worse with deep inspiration, and shortness of breath. Vital signs were all within normal limits. A CT angiogram revealed a Stanford Type B aortic dissection. She was transferred to our facility for higher level of care. On arrival she continued to be hemodynamically stable with systolic blood pressures ranging from 100 to $120 \mathrm{~mm} \mathrm{Hg}$. Vascular Surgery consultation was obtained and decision was made to continue with medical management at that time. Mean arterial pressure remained within goal without use of intravenous nicardipine. The patient was transferred to the surgical intensive care unit for continued observation and pain control with intravenous narcotics.

Approximately 12 hours after admission she developed excruciating back and chest pain radiating to her left arm and left side of her neck, which was no longer responsive to pain medications. At this time, she was afebrile with blood pressure ranging from 100 to $139 \mathrm{~mm} \mathrm{Hg}$ systolic, heart rate 59 to 88 beats per minute, respiratory rate of 12 breaths per minute and oxygen saturation of $90 \%$ to $97 \%$. An emergent $\mathrm{CT}$ angiography of the chest, abdomen, and pelvis was performed which reconfirmed the type B dissection originating $1.5 \mathrm{~cm}$ from the left subclavian take off and extending to the proximal left common iliac artery (-Figs. 1a, b and $\mathbf{2}$ ). Importantly, a new hyperdense foci in the distal aortic arch suggestive of active contrast extravasation was detected (-Figs. 3a, b). On concurrent echocardiogram the aortic root measured $41.6 \mathrm{~mm}$ at the sinus of Valsalva with flattening at the sinotubular junction (measuring $36.8 \mathrm{~mm}$ ).

The patient was taken emergently to the operating room. Intraoperatively, the type $\mathrm{B}$ dissection was noted to have expanded to the level of the subclavian orifice from a few hours prior on imaging and rupture of the dissection was confirmed. Two Bolton Relay thoracic stent grafts were used, the proximal one measuring $26 \mathrm{~mm}$ and $10 \mathrm{~cm}$ long, the distal one measuring 28 to $24 \mathrm{~mm}$ tapered measuring $20 \mathrm{~cm}$ long with $5 \mathrm{~cm}$ of overlap. The entire stented region started just distal to the takeoff of the left common carotid and extended to $2 \mathrm{~cm}$ above the takeoff of the celiac axis. The procedure was uncomplicated. The estimate blood loss 

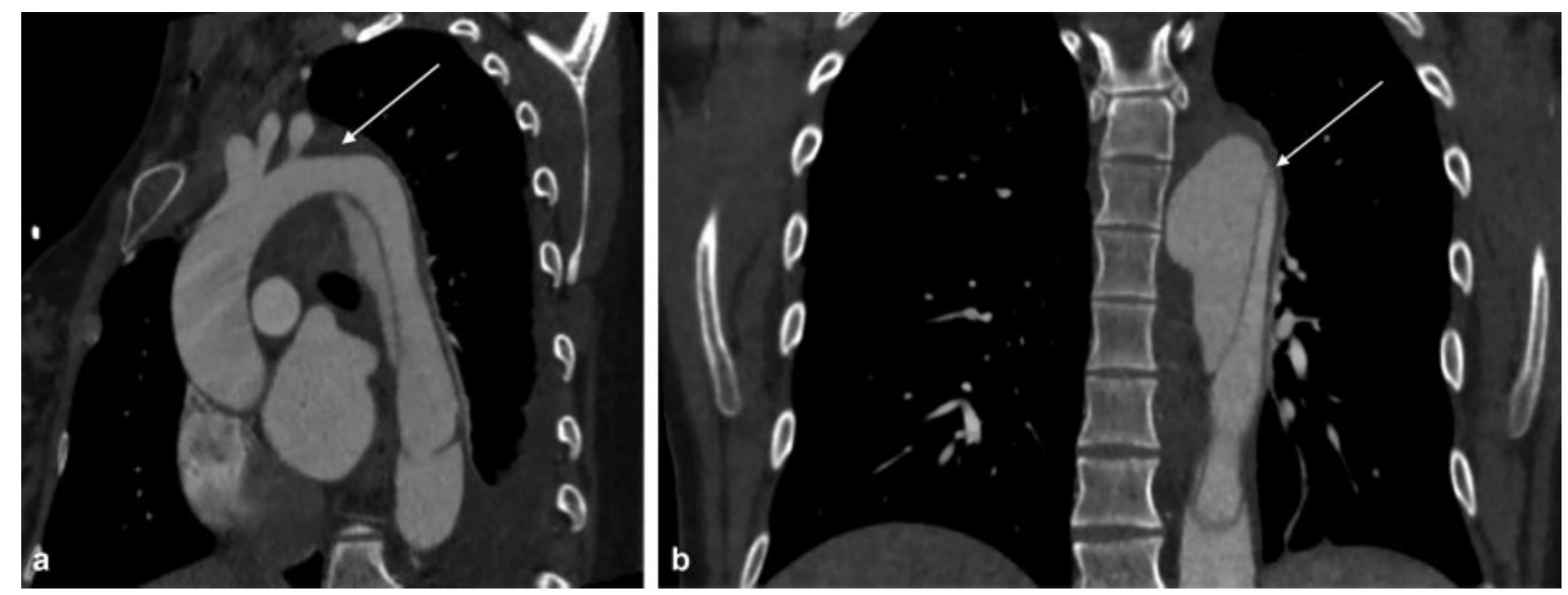

Fig. 1 CT angiography of chest-(a) sagittal and (b) coronal-demonstrating Stanford Type B aortic dissection originating in the distal aortic arch (arrows).

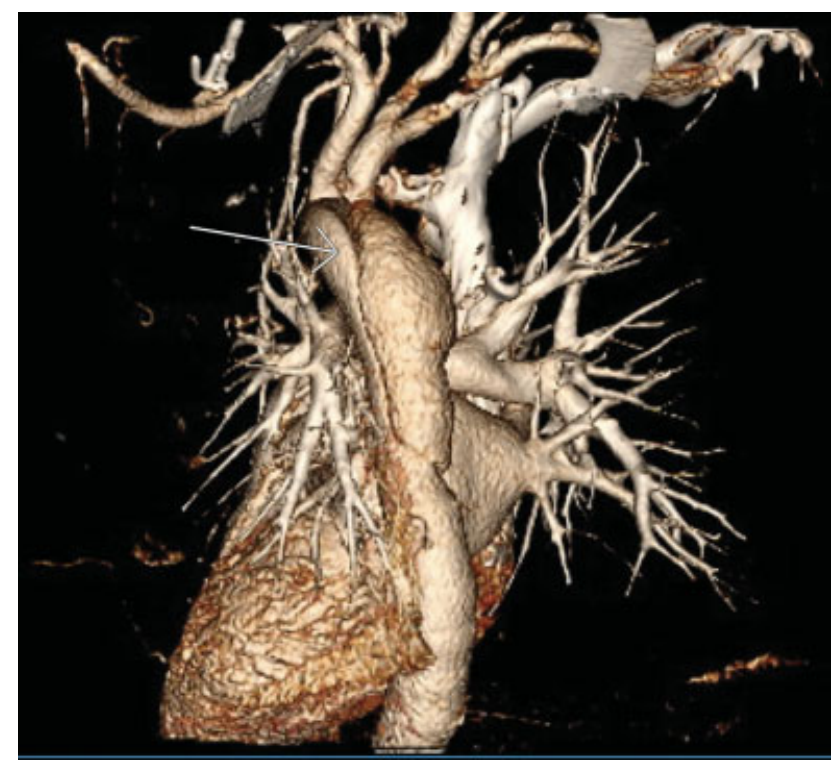

Fig. $2 \mathrm{CT}$ angiography 3D reconstruction demonstrating dissection of distal aortic arch/proximal descending aorta (arrow) originating just distal to subclavian artery take-off. was $100 \mathrm{~mL}$ and one unit of packed red blood cells was given.

Repeat CT angiography of the whole aorta on postoperative day 2 showed patent stent extending to the true lumen of supraceliac proximal abdominal aorta with exclusion of distal aortic arch-proximal descending aorta aneurysm without evidence of perigraft contrast extravasation. Postoperative course was uncomplicated and she was discharged home on postoperative day 10 with follow-up with vascular surgery. She was continued on metoprolol. At her 6-week postpartum visit she was asymptomatic and was strongly counseled to avoid future pregnancy. She elected to undergo laparoscopic bilateral tubal ligation which was performed several months later without complications.

\section{Discussion}

This case highlights the ongoing controversy over how pregnant patients with MFS and an aortic root between $40 \mathrm{~mm}$ and $45 \mathrm{~mm}$ should be managed. Our patient was managed in accordance with the most recent consensus guidelines, including those published by the European
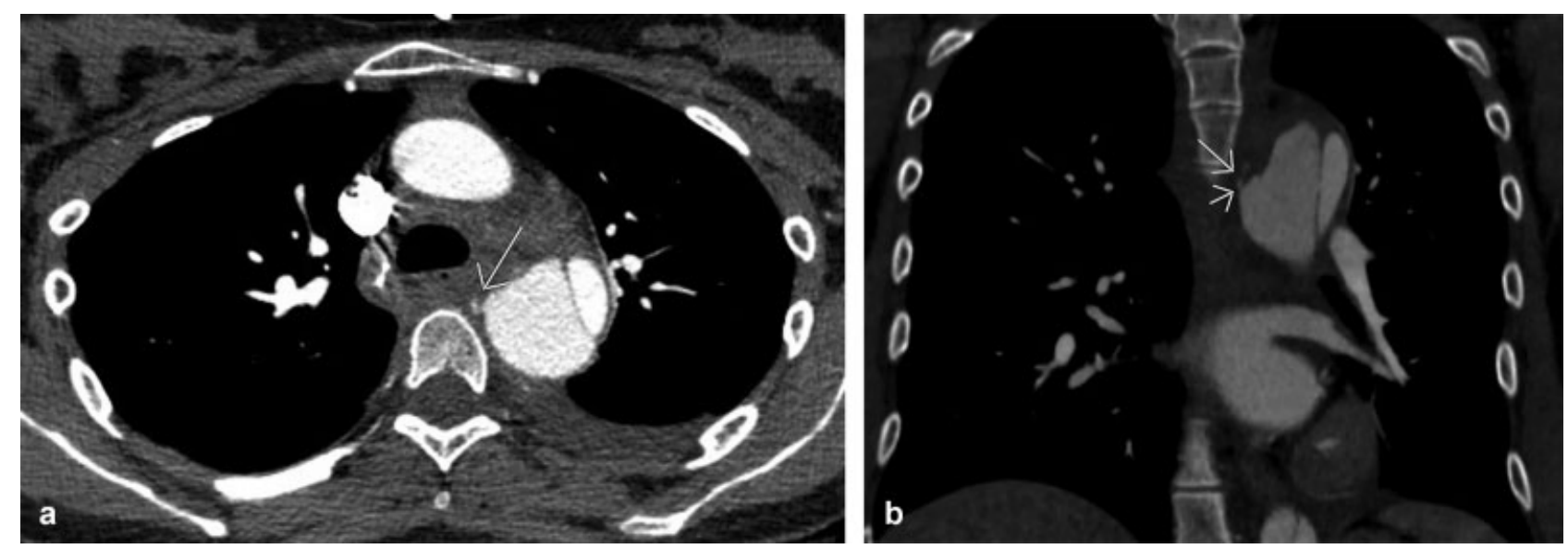

Fig. 3 CT angiography-(a) axial and (b) coronal-images of region suspicious for contrast extravasation (arrows) along proximal descending aortic arch dissection. 
Society of Cardiology and the Canadian Cardiology Society, which agree that pregnancy is not recommended if the aortic root is $>45 \mathrm{~mm}$ (or $>40 \mathrm{~mm}$ in the presence of rapidly enlarged dilation or family history, neither of which our patient had). ${ }^{17,18}$ On the other hand, the 2010 American College of Cardiology guidelines recommend against pregnancy for MFS patients if the aortic root is $>40 \mathrm{~mm}$, or for prophylactic repair prior to conceiving. ${ }^{12}$ Lack of consensus on aortic root diameter and risk of dissection is confounded by the fact that standardization of aortic root measurements is lacking. Some experts advocate for aortic root measurement at the sinus of Valsalva, claiming that this is the segment that dilates first and is most prone to dissection in MFS. ${ }^{12,21}$ However, dilation of the root at the level of the sinotubular junction, or extending to the ascending aorta, is known to portend a poor prognosis and so these measurements should also be considered. ${ }^{22}$ In our case, the patient was felt to be lower risk because of stable measurements $<45 \mathrm{~mm}$ at the sinus of Valsalva and $<40 \mathrm{~mm}$ at the sinotubular junction.

In the primary literature on MFS in pregnancy, some authors describe measurements of the aortic root being taken at the sinus of Valsalva ${ }^{11,13}$ while others do not specify. ${ }^{8}$ Consensus guidelines on management of thoracic aortic disease in MFS do not specify how or where the aortic root should be measured. ${ }^{12,17}$ Furthermore, studies that have reported echocardiographic measurements of aortic root at the Sinus of Valsalva for MFS in pregnancy also differ in convention of timing of measurement, with some taken during end-diastole ${ }^{16}$ and others during mid-systole. ${ }^{11}$ Whether to measure leading-edge to leading-edge, outeredge to outer-edge, versus inner-edge to inner-edge differs between the pediatric and adult literature and varies depending on mode of imaging (CTVs. echo) and has changed over time. ${ }^{16,17,21}$ These discrepancies are important as they may cause variations of up to $4 \mathrm{~mm}$, which could significantly change counseling. In our case, echocardiographic measurements were obtained during diastole measuring the widest portion of the right coronary to left coronary sinus from leading-edge to leading-edge in the parasternal long-axis view in accordance with 2015 American Society of Echocardiography guidelines. ${ }^{23}$ We advocate for multiple, standardized measurements along various segments of the aorta including at the sinus of Valsalva, the sinotubular junction, and the ascending aorta with consistency over time for better comparison and prognostication.

The lack of standardization and consensus regarding aortic root diameter and risk of dissection and other aortic complications underscores the importance of identifying all potential risk factors when counseling and managing pregnant women with MFS. For example, the rate of aortic root growth is an important consideration and has been shown to be a poor prognostic indicator. ${ }^{22}$ While our patient did not exhibit growth at the sinus of Valsalva, she did exhibit some extension along the length of the root, consistent with effacement at the sinotubular junction $(3.12 \mathrm{~mm}$ in the first trimester to $3.76 \mathrm{~mm}$ in the second trimester and postpartum). While these measurements remained $<4 \mathrm{~cm}$, it is known that extension of the aneurysmal dilation beyond the sinus is associated with worse outcomes. ${ }^{22}$ Thus, providers should be careful to measure multiple portions of the root and to pay attention to changes other than just at the sinus of Valsalva.

In an attempt to standardize aortic measurements, some experts factor in body surface area, such as the Aortic Size Index (ASI). Davies et al first described this measurement of relative aortic size (aortic root diameter $[\mathrm{mm}] /$ body surface area $\left.\left[\mathrm{m}^{2}\right]\right)$ in predicting rupture of thoracic aortic aneurysms in nonpregnant adults. ${ }^{24}$ The utility of ASI measurements was particularly evident in a retrospective review in a Japanese cohort which found a high rate of complications in pregnant women with aortic root $<40 \mathrm{~mm}$ and it was hypothesized that this is secondary to small stature. ${ }^{25}$ The authors concluded that an ASI score rather than an absolute diameter size is important in determining risk and that an ASI of $>25 \mathrm{~mm} / \mathrm{m}^{2}$ was predictive of aortic complications in their population. Our patient's ASI in the first trimester was $21.5 \mathrm{~mm} / \mathrm{m}^{2}$. Further research is needed to validate this novel measurement tool in pregnant patients with MFS.

Our case highlights the fact that the postpartum period remains a high-risk period for women with MFS. In a literature review that identified 88 cases of aortic dissection complicating pregnancy in MFS patient, 26\% of cases occurred postpartum. ${ }^{10}$ Many of these cases occurred in women whose aortic root was $<40 \mathrm{~mm}$. Consistently, the literature suggests that the postpartum period is the second most frequent time for aortic complications to occur, after the third trimester. ${ }^{10,26,27}$ ED physicians should be alerted to the fact that the postpartum period remains a significantly elevated risk time period for MFS patients. In a large database study of MFS patients followed longitudinally, 7 aortic dissections occurred out of 227 pregnancies, 6 of which happened postpartum. ${ }^{28}$ Consideration for close follow-up postpartum with repeat imaging should be given, even in cases where the aortic root is not dilated.

Aneurysms and dissections can occur anywhere along the aorta in pregnant MFS patients, as evidence by our case. Predicting Type B (distal) aortic dissections poses a particular dilemma. According to one large international registry database study, $36 \%$ of dissections in nonpregnant MFS patients are Type $B .^{29}$ Based on less robust data, this number is cited to be $\sim 33 \%$ in the MFS in pregnancy literature. ${ }^{10,26}$ Because MFS is classically associated with Type A dissection, the focus has been on measuring the aortic root in predicting risk of complications; however, there is insufficient evidence to conclude whether or not aortic root dilation is also predictive of Type $\mathrm{B}$ dissection. In a literature review of 1,112 pregnancies in MFS patient from 2005 to 2015, Kim et al found no clear association between aortic diameter and type of dissection. ${ }^{10}$ Minsart et al prospectively followed 21 pregnancies in women with MFS and reported two complications, both of which were Type B dissections in women with an aortic root $<40 \mathrm{~mm}$. ${ }^{16}$ Providers should be alerted to the fact that a substantial minority of MFS pregnancy related complications will occur more distally along the aorta and, therefore, the entire aorta should be imaged prior to 
pregnancy or as early on as possible. Serial measurements of the descending and abdominal aorta are costly and there is no established cutoff at which a patient is at increased risk; however, this may be considered in patients who have aneurysmal dilation of distal portions of the aorta at baseline. Our patient did not exhibit descending arch aneurysm in her prepregnancy CT angiogram; however, the abdominal aorta was not imaged. Whole aorta screening imaging was not repeated given the lack of early dilation but perhaps would be warranted, at least early postpartum once radiation exposure no longer poses a risk to the fetus.

Reports of aortic rupture complicating a pregnancy in MFS are rare. In a recent literature review of all reported cases of MFS in pregnancy from 2005 to 2015 including 1,112 pregnancies, there were only five cases of aortic rupture/ tamponade identified, 3 of which led to maternal deaths. ${ }^{10}$ Typically, uncomplicated Type B dissections are managed medically, but pregnant MFS patients have different pathophysiology and risk factors that may require a different approach. The poor outcomes in medically managed Type B dissections in MFS patients has led some to argue for early endovascular repair. ${ }^{30}$ The timely diagnosis and repair likely accounted for the good outcome in our patient's case.

\section{Conclusion}

We described a case of aortic rupture following a Type B aortic dissection in a postpartum patient with MFS with aortic root (at the sinus of Valsalva) $<4.5 \mathrm{~cm}$. The timely diagnosis and prompt intervention by a multidisciplinary team led to a favorable outcome. Patient and health care provider education, close surveillance and frequent monitoring, preferably of the whole aorta, may be warranted given that the Type $B$ dissections may not be visualized by the routine transthoracic echocardiographic assessment of the aortic root. Early surgical repair of Type B aortic dissection in postpartum MFS patients should be considered. There is a need to standardize how and where the aorta is measured in patients with MFS to assess risk of complications during pregnancy.

\section{Conflict of Interest/Disclosure Statement}

There were no sources of funding or financial support for this study. The authors report no conflicts of interest.

\section{Acknowledgments}

We would like to thank Deniz Urgun, MD, with the Department of Radiology at the University of California, Irvine, for her assistance in interpreting and selecting the radiographic images for this manuscript.

\section{References}

1 Judge DP, Dietz HC. Marfan's syndrome. Lancet 2005;366 (9501):1965-1976

2 Collins MJ, Dev V, Strauss BH, Fedak PW, Butany J. Variation in the histopathological features of patients with ascending aortic aneurysms: a study of 111 surgically excised cases. J Clin Pathol 2008;61(04):519-523
3 Trotter SE, Olsen EG. Marfan's disease and Erdheim's cystic medionecrosis. A study of their pathology. Eur Heart J 1991;12 (01):83-87

4 Manalo-Estrella P, Barker AE. Histopathologic findings in human aortic media associated with pregnancy. Arch Pathol 1967;83 (04):336-341

5 Goland S, Elkayam U. Cardiovascular problems in pregnant women with Marfan syndrome. Circulation 2009;119(04): 619-623

6 Goland S, Elkayam U. Pregnancy and Marfan syndrome. Ann Cardiothorac Surg 2017;6(06):642-653

7 Rossiter JP, Repke JT, Morales AJ, Murphy EA, Pyeritz RE. A prospective longitudinal evaluation of pregnancy in the Marfan syndrome. Am J Obstet Gynecol 1995;173(05):1599-1606

8 Pyeritz RE. Maternal and fetal complications of pregnancy in the Marfan syndrome. Am J Med 1981;71(05):784-790

9 Goland S, Barakat M, Khatri N, Elkayam U. Pregnancy in Marfan syndrome: maternal and fetal risk and recommendations for patient assessment and management. Cardiol Rev 2009;17(06): 253-262

10 Kim SY, Wolfe DS, Taub CC. Cardiovascular outcomes of pregnancy in Marfan's syndrome patients: a literature review. Congenit Heart Dis 2018;13(02):203-209

11 Donnelly RT, Pinto NM, Kocolas I, Yetman AT. The immediate and long-term impact of pregnancy on aortic growth rate and mortality in women with Marfan syndrome. J Am Coll Cardiol 2012;60 (03):224-229

12 Hiratzka LF, Bakris GL, Beckman JA, et al; American College of Cardiology Foundation/American Heart Association Task Force on Practice Guidelines; American Association for Thoracic Surgery; American College of Radiology; American Stroke Association; Society of Cardiovascular Anesthesiologists; Society for Cardiovascular Angiography and Interventions; Society of Interventional Radiology; Society of Thoracic Surgeons; Society for Vascular Medicine. 2010 ACCF/AHA/AATS/ACR/ASA/SCA/SCAI/ SIR/STS/SVM guidelines for the diagnosis and management of patients with thoracic aortic disease: a report of the American College of Cardiology Foundation/American Heart Association Task Force on Practice Guidelines, American Association for Thoracic Surgery, American College of Radiology, American Stroke Association, Society of Cardiovascular Anesthesiologists, Society for Cardiovascular Angiography and Interventions, Society of Interventional Radiology, Society of Thoracic Surgeons, and Society for Vascular Medicine. Circulation 2010;121(13): e266-e369

13 Meijboom LJ, Vos FE, Timmermans J, Boers GH, Zwinderman AH, Mulder BJ. Pregnancy and aortic root growth in the Marfan syndrome: a prospective study. Eur Heart J 2005;26(09): 914-920

14 Omnes S, Jondeau G, Detaint D, et al. Pregnancy outcomes among women with Marfan syndrome. Int J Gynaecol Obstet 2013;122 (03):219-223

15 Kuperstein R, Cahan T, Yoeli-Ullman R, Ben Zekry S, Shinfeld A, Simchen MJ. Risk of aortic dissection in pregnant patients with the Marfan syndrome. Am J Cardiol 2017;119(01):132-137

16 Minsart AF, Mongeon FP, Laberge AM, Morin F, Dore A, Leduc L. Obstetric and cardiac outcomes in women with Marfan syndrome and an aortic root diameter $\leq 45 \mathrm{~mm}$. Eur J Obstet Gynecol Reprod Biol 2018;230:68-72

17 Erbel R, Aboyans V, Boileau C, et al; ESC Committee for Practice Guidelines; The Task Force for the Diagnosis and Treatment of Aortic Diseases of the European Society of Cardiology (ESC). 2014 ESC Guidelines on the diagnosis and treatment of aortic diseases: document covering acute and chronic aortic diseases of the thoracic and abdominal aorta of the adult. Eur Heart J 2014;35 (41):2873-2926

18 Boodhwani M, Andelfinger G, Leipsic J, et al; Canadian Cardiovascular Society. Canadian Cardiovascular Society position 
statement on the management of thoracic aortic disease. Can J Cardiol 2014;30(06):577-589

19 Regitz-Zagrosek V, Roos-Hesselink JW, Bauersachs J, et al; ESC Scientific Document Group. 2018 ESC Guidelines for the management of cardiovascular diseases during pregnancy. Eur Heart J 2018;39(34):3165-3241

20 Loeys BL, Dietz HC, Braverman AC, et al. The revised Ghent nosology for the Marfan syndrome. J Med Genet 2010;47(07): 476-485

21 Wright M, Connolly H. Management of Marfan syndrome and related disorders. In: Post T, ed. UpToDate Website. https://www. uptodate.com/contents/management-of-marfan-syndrome-andrelated-disorders. Accessed April 13, 2019

22 Roman MJ, Rosen SE, Kramer-Fox R, Devereux RB. Prognostic significance of the pattern of aortic root dilation in the Marfan syndrome. J Am Coll Cardiol 1993;22(05):1470-1476

23 Goldstein SA, Evangelista A, Abbara S, et al. Multimodality imaging of diseases of the thoracic aorta in adults: from the American Society of Echocardiography and the European Association of Cardiovascular Imaging: endorsed by the Society of Cardiovascular Computed Tomography and Society for Cardiovascular Magnetic Resonance. J Am Soc Echocardiogr 2015;28(02):119-182

24 Davies RR, Gallo A, Coady MA, et al. Novel measurement of relative aortic size predicts rupture of thoracic aortic aneurysms. Ann Thorac Surg 2006;81(01):169-177
25 Katsuragi S, Ueda K, Yamanaka K, et al. Pregnancy-associated aortic dilatation or dissection in Japanese women with Marfan syndrome. Circ J 2011;75(11):2545-2551

26 Smith K, Gros B. Pregnancy-related acute aortic dissection in Marfan syndrome: a review of the literature. Congenit Heart Dis 2017;12(03):251-260

27 Elkayam U, Ostrzega E, Shotan A, Mehra A. Cardiovascular problems in pregnant women with the Marfan syndrome. Ann Intern Med 1995;123(02):117-122

28 Roman MJ, Pugh NL, Hendershot TP, et al; GenTAC Investigators Dietz Harry C, Habashi Jennifer, Prakash Siddharth K, Maslen Cheryl L, Song Howard K, Bavaria Joseph E, Milewski Karianna, Weinsaft Jonathan W, McDonnell Nazli, Asch Federico M, Tolunay H Eser, Desvigne-Nickens Patrice, Tseng Hung, Kroner Barbara L. Aortic complications associated with pregnancy in Marfan syndrome: The NHLBI National Registry of Genetically Triggered Thoracic Aortic Aneurysms and Cardiovascular Conditions (GenTAC). J Am Heart Assoc 2016;5(08):e004052

29 de Beaufort HWL, Trimarchi S, Korach A, et al. Aortic dissection in patients with Marfan syndrome based on the IRAD data. Ann Cardiothorac Surg 2017;6(06):633-641

30 Umaña JP, Miller DC, Mitchell RS. What is the best treatment for patients with acute type $B$ aortic dissections-medical, surgical, or endovascular stent-grafting? Ann Thorac Surg 2002;74(05): S1840-S1843, discussion S1857-S1863 